\title{
Beyond pessimism: The dialectic of promise and complexity in genomic research
}

\author{
MICHAEL ARRIBAS-AYLLON ${ }^{1}$
}

\begin{abstract}
How should we reflect upon the last 10 years since the completion of the human genome? One dominant response from within the humanities and the social sciences is to cast these events within a dialectic of promise and disappointment. Indeed, this contrast would seem to hold if we take Clinton's historic announcement as our point of departure. I choose an alternative departure: not in the rhetoric of press releases but from scientists' ambivalent accounts of complexity. Perhaps a dialectic of promise and complexity is a less pessimistic (but no less sceptical) way of reflecting on what has happened in the last 10 years. In this paper, I focus on two aspects of societal change within the 'urban zone': the rise of population-based biobanking and the marketisation of genetic susceptibility testing. Both developments are driven by the promise that genomic research will lead to new ways to 'prevent, diagnose, treat and cure disease'. However, genomic knowledge also reveals a level of complexity that has led to unprecedented scale in the production of granular information. In the last 10 years we have seen that traditional bioethics has struggled to cope with this scale. In the era of high-throughput sequencing and personal genomics, we have also seen that translating complexity into benefits for the health consumer is controversial. Arguably, ethical principles do not capture the subtle differences between predictive and susceptibility testing, and that more empirical research is needed to understand how people perceive and communicate complex risk information.
\end{abstract}

\section{Introduction}

When politicians are given the task of showcasing scientific achievements, optimism and hyperbole are often key ingredients of telling a good story. This was certainly the case when, on 25 June 2000, President Clinton announced "the completion of the first survey of the entire human genome". ${ }^{2}$ Leaving aside the familiar tropes of blueprints, maps and codes, on that day a central promise was made: "Decoding the human genome will lead to new ways to prevent, diagnose, treat, and cure disease". 3 Ten years on, the task of this special issue is to reflect on the extent to which this promise has been realised and to consider how the 'societal landscape of genomics' has changed in this period. While holding politicians to their promises is prone to disappointment, I want to suggest that we look elsewhere to undertake this task of historical reflection. Rather than in the rhetoric of press releases or in the expectations of 'completion', I want to begin with scientists' ambivalent accounts of what that completed sequence contained: the complexity of DNA.

In February 2001, an article co-authored by Craig Venter and 275 other contributors appeared in Science entitled: 'The sequence of the human genome'. ${ }^{4}$ This article

(C) ESRC Genomics Network.

Genomics, Society and Policy, Vol.6, No.2 (2010) ISSN: 1746-5354 
offered a sobering description of the characteristics of the human genome sequence and a more circumspect account of promise. Actually, the 'major surprise' that the human genome contained far fewer genes than expected $(23,000-26,000$ rather than the predicted 50,000 - to 140,000) was not an indication of the reduced complexity of the human genome; quite the reverse. It meant that complexity was virtually hidden among the diversity of non-linear protein-protein interactions. The provisional map of the human sequence provided yet another blow to the 'gene for' paradigm: "This dynamic system ... has many ways to modulate activity, which suggests that definition of complex systems by analysis of single genes is unlikely to be entirely successful," wrote Venter et al. ${ }^{5}$ This cautious statement does not rule out the possibility that rare, highly penetrant, genes will be implicated in the pathogenesis of some diseases, but it does strengthen the hypothesis that many 'common' diseases are polygenic and multifactorial: "Thus, there are no 'good' genes or 'bad' genes, but only networks that exist at various levels and at different connectivities, and at different states of sensitivity to perturbation." "In contrast to the kind of unqualified promise made a year earlier, here we see that scientists' engage in highly moderated forms of promising based not on the imperative of translation (i.e. curing or preventing disease, etc.) but in overcoming a set of technical problems that have suddenly come into view: "The next steps are clear: We must define the complexity that ensues when this relatively modest set of about 30,000 genes is expressed."7

Since Clinton's historic announcement, we have seen an increasing pessimism towards the rhetoric of (genomic) promise. I want to suggest that rather than dwelling on a dialectic of promise and disappointment, we could look at the last decade less pessimistically (but no less sceptically) in terms of a dialectic of promise and complexity. By this I mean that we could examine the societal landscape of genomics in terms of various attempts to define, map and exploit genomic complexity; we could trace different and competing versions of complexity that engender their own forms of promise: future health and wellbeing, autonomy and security, solidarity and social justice; public trust and participation.

Complexity is, admittedly, an opaque term that can mean different things to different people. In the context of genomics, 'complexity' is the probabilistic uncertainty arising from multigenic and multifactorial models of disease risk. The uncertainty arising from genomic explanations is not simply a matter of identifying all the known or hidden factors that contribute to disease, but also recognising the non-linear and stochastic nature of gene-gene and gene-environment interactions. The uncomfortable (re)discovery of complexity is indeed related to the promise of broadening the scope of genomic research to investigate the underlying biology of 'common complex' diseases. The dialectic of promise and complexity proposed here is one that examines the ethical and social challenges arising from the enlargement of promise, especially when complexity is denied in the marketisation of risk prediction or when complexity increases demands for the collection of human DNA material.

This article examines the last decade of genomics in the context of the 'urban zone' the application of genomics in the domain of contemporary healthcare, education and 
research. It is well beyond the scope of this article to offer a complete map of the urban zone. Instead, I will examine the interplay of promise and complexity in two main areas: i) population-based biobanks as data repositories for researching genomic complexity; and ii) genetic susceptibility testing as efforts to translate complexity into risk prediction.

\section{The rise of population-based biobanking}

The collection of genetic data on populations and families for medical research has existed for more than 30 years. These 'genetic databases' were at first relatively uncontroversial and small-scale. Since the Human Genome Project (HGP), however, the rise of population-based biobanking has become a controversial enterprise. The understanding that many common diseases such as cancer, heart disease and dementia are 'complex' means that research requires an ever-increasing volume of 'granular' (i.e. detailed) information. Genome-wide association studies (GWAS), for example, require much greater statistical power to identify both common and rare variants. This involves recruiting much larger samples of DNA - the bigger the better. In this sense, biobanks supply the essential capital to meet the increasing scale of population-based research.

The new generation of biobanks operates on a prospective basis, involving large-scale collections of DNA and personal medical information being held for long periods of time and for unspecified purposes. Despite the uncertainties of retaining this kind of sensitive information, they hold considerable promise for explaining the complex aetiology of common diseases, for improving future health for all, and for providing substantial economic benefits for their investors. Not surprisingly, in the last 10 years there has been considerable debate about the challenges that biobanks face in gaining consent, legitimacy and trust. ${ }^{8}$

It is not uncommon that when a practice becomes 'controversial' its meaning also becomes unstable. But controversies are also productive in that they provide an opportunity to respecify and stabilise ambiguous meanings. For instance, there have been disagreements in the literature about the definition of biobanks (e.g. whether it should be replaced by the term 'genetic database'); they have been connected to a whole range of activities (e.g. research in common complex diseases, pharmacogenetics, rare genetics diseases, oncology and stem cells, etc.); they are embedded in numerous institutions (hospitals, universities, pharmaceutical companies and charities); and their global development has been treated with suspicion (initiatives have appeared in the UK, EU, Quebec, USA, China, Singapore, Taiwan, Japan and Australia). The ambivalent relationship between public and private investment has also cast doubt on the legitimacy of biobanks: potentially they are a public good but they require commercial investment to actualise this good. To add further grounds for scepticism, recent historical events in the UK have eroded public trust in science (e.g. mad-cow disease, the GM debate, leaked climate change emails) triggering a 'crisis in trust'. 
In the current climate of public scepticism towards science and governance, the developers and managers of biobanks face many challenges in establishing frameworks that can guarantee data security and win public trust. Such problems have thrown into relief the methods of consulting publics and communities and the various programmes designed to foster 'active citizenship' and 'participation' to remedy this situation. Some commentators have argued that public engagement projects may actually limit debate and be perceived by publics as mechanisms for manufacturing consent. ${ }^{10}$ In the UK, there are particular concerns that public engagement has been treated as a kind of 'risk management strategy' rather than genuinely involving publics in issues of management. ${ }^{11}$ Furthermore, the ethical and political aspects of biobanking have exposed the instability of the category 'the public', illustrating the degree to which the lingering belief in 'knowledge deficits' constitute 'the public' as ignorant, irrational, selfish and ambivalent. ${ }^{12}$

An issue that has attracted a great deal of attention in the literature is the process of securing informed consent from individuals who donate tissue samples and personal information. The events surrounding the Icelandic Health Sector Database in the late 1990s illustrated the problems arising from presumed consent: that the burden of responsibility was placed on individuals to opt out before a certain period to prevent their medical records from being included in the Database. In many ways, the controversy demonstrated how not to develop a biobank. ${ }^{13}$ Over the last 10 years of biobank development there has been increasing involvement of bioethicists whose expertise in policy-relevant knowledge has provided additional layers of oversight. But rather than strengthening governance, there have been growing concerns that ethical frameworks produce bureaucratic overload, duplication of paperwork, and performative accountability. ${ }^{14}$ Furthermore, traditional concepts of bioethics such as informed consent seem to impede research activity because the prospective nature of biobanking requires provision for multiple researchers over long periods of time; seeking re-consent can be time-consuming and expensive, while the possibility of refusals or non-responses can undermine the integrity of research. Some have even claimed that continual requests for consent might actually foster negative attitudes towards biomedical research. ${ }^{15}$ Williams argues that in the UK experience of largescale biobanking, the focus on informed consent is a distraction from political questions about the organisation of medical research or the accumulation of state power. ${ }^{16}$ In the UK, ethical frameworks have opted for 'open consent' which affords greater flexibility between research and individual autonomy. However, Tutton et al. have warned that open consent restricts the rights of participants (and their relatives) to withdraw from future research. ${ }^{17}$

The debate on whether the concept of informed consent captures the relevant tensions between the micro and the macro is exacerbated by the enormous scale of genomic research. Knopper and Chadwick have argued that "the increase in population-based genetic research has led to calls for rethinking the paramount position of the individual in ethics". ${ }^{18}$ The complexity of the human genome warrants a new conceptualisation of ethics based on principles of reciprocity, mutuality, solidarity, citizenry and universality. According to this view, people have a duty to participate in 
biobanks as resources that will benefit future generations of society. The universality implied in such notions of public and future good outweighs autonomy as the ultimate arbiter. However, debates about whether new approaches to ethical reasoning are needed are further problematised by the pivotal role that biobanks play in the bioknowledge economy and the globalisation of bioinformation. ${ }^{19}$ Biobanks can be seen as collection points for the harvesting of raw material in the production of biovalue. ${ }^{20}$ The biological characteristics or 'potentials' of populations are treated as raw materials for economic exploitation. The precise nature of these raw materials is that they themselves are unstable or 'metastable' boundary objects - in different domains and to different actors they can be 'things', 'people' or segments of 'information'. Whether human tissue is the property of the donor (and of potential commercial value) or the property of those whose labour is invested (thereby creating commercial value) has been debated in case law and legal theory. ${ }^{21}$ The key tension is that if commercial parties stand to profit from human tissue, should individuals from whom the tissue is taken be granted property rights to share in these profits? The argument that commercial investment in research on common disease is the necessary trade-off for significant future benefits is indicative of the way in which universal principles of the public good tend to surpass individual rights.

Biobanks have not only attracted ethical and political controversy, but they are also linked in complex ways to the politics of (collective) identity and race. Since the HGP, some commentators have expressed concerns about the biological reinscription of race. ${ }^{22}$ Rose argues that "race now signifies an unstable space of ambivalence" between molecular biology and socio-political identification. ${ }^{23}$ Despite the overwhelming genetic similarity shared across the spectrum of human difference, since the HGP it is the minor variations (e.g. single-nucleotide polymorphism - SNPs and haplolytes) that have been significantly correlated with disease susceptibility in different populations. Even notions of population have been constructed in terms of whether their purported genetic homogeneity or heterogeneity are sources of 'exchange value'. ${ }^{24}$ The portrayal of Iceland as genetically isolated and homogenous formed part of the promissory value of the Health Sector Database. ${ }^{25}$ However, a different identity politics has been played out in the USA and the UK, where issues of ethnic diversity are more prominent. In these countries, tensions between scientific methodology and social inclusion have emerged in relation to research on common complex diseases. Smart et al. cite evidence that a minority of researchers who worked in UK biobanks justified excluding ethnic and racial minority groups on methodological grounds (i.e. where race and ethnicity are potentially 'confounding factors' in homogenous populations). ${ }^{26}$ In the US, similar concerns have led to initiatives to establish biobanks around the needs of specific racial/ethnic groups (e.g. African-Americans and Orthodox Jews). With the potential to identify different disease susceptibilities among different populations, the issue is not whether genomic research will reactivate further stigma and intolerance but how new rationalities and technologies of power over life will govern human differences. ${ }^{27}$ 


\section{Genetic susceptibility testing}

If prospective, large-scale collections of genetic and non-genetic information are one response to genomic complexity, then another is the translation of these raw materials into clinically relevant risk information. The HGP demonstrated both the means and the possibility of identifying many thousands of genes of small to moderate effect. It confirmed scientists' suspicions that many common diseases are in fact multifactorial, comprising gene-gene interactions as well as gene-environment interactions. This is very different from the kind of genetic risk commonly associated with presymptomatic predictive testing for Mendelian conditions such as Huntington's disease, cystic fibrosis and Fragile $\mathrm{X}$ syndrome. In the absence of a single dominant gene, genetic risk is essentially probabilistic and thus calculated on a delicate balance of uncertainties. And yet, in the last 10 years, there has been a massive increase in the marketisation of genetic susceptibility testing, the central promise of which is to predict and prevent disease. These developments are highly controversial for two reasons: the uncertainty of probabilistic risk information seriously undermines the validity of such tests, and the predictive or diagnostic inferences of such information may have an adverse psychosocial impact on individuals and families. The task is to consider the ethical and social differences between strongly and weakly predictive testing.

The social, ethical, legal and psychosocial aspects of genetic testing have been debated since the 1970s. A dominant style of professional ethical reasoning is the conceptualisation of ethical principles and disease exemplars. During the 1980s and 1990s, for example, the extreme scenario of Huntington's disease (HD) provided an ideal exemplar for the development of ethical policy. Boddington and Hogben have argued that using HD as an exemplar stresses "a difference in degree of seriousness" which "translates into a substantive difference of kind in justifying ethical argument". ${ }^{28}$ They query whether the selection of other candidate conditions may have led to different policy conclusions. We could say that ethical policy for genetic testing has been influenced by the mode of inheritance; that is to say, HD was cast as an extreme case that exemplified the 'geneticisation' of ethical reasoning and the privileging of genetic factors over other aspects of a disease condition. Case exemplars have also made an impact on ethical discussions in the social sciences. Novas and Rose use the example of HD to argue persuasively that rather than inducing fatalism and resignation, predictive testing is linked to the creation of new forms of subjectivity and 'genetic responsibility'. ${ }^{29}$ Rather than viewing genetic knowledge as objectifying and repressive, they argue that practices of 'genetic subjectification' (i.e. the creation of subjects through knowledge/power relations) are in fact aligned with the norms of advanced liberalism, which explains why principles such as autonomy are frequently used in ethical discussions. They propose that genetic knowledge is operating in a political field where subjects are constructed as autonomous, prudent, responsible and self-actualising.

So what kind of exemplar might we use in the case of genetic susceptibility testing? One candidate for discussion is Alzheimer's disease (AD). For the last 20 years, there 
has been consensus among researchers that rare autosomal dominant genes are strongly associated with the 'early onset' form of dementia. However, in 1993 the apolipoprotein $\mathrm{E}$ (ApoE) $\varepsilon 4$ allele on chromosome 19 was found to be associated with an increased risk for the common 'late onset' form of $\mathrm{AD} .{ }^{30}$ The presence of $\varepsilon 4$ increases risk of AD up to 15-fold compared to other ApoE polymorphisms. The aetiology of $\mathrm{AD}$ shows that susceptibility testing is different from predictive testing in that risk information is relevant to a much larger population but is much less certain than predictive testing. ${ }^{31}$ Given that there are no treatment options for $\mathrm{AD}$, professionals have cautioned against the routinisation of susceptibility testing in presymptomatic individuals unless to confirm an AD diagnosis. ${ }^{32}$ The ethical differences between susceptibility and predictive testing may appear to be differences of degree rather than kind if we apply principles as our bench mark. However, as Evans has noted, principlism is "a method that takes the complexity of actually lived moral life and translates this information into four scales by discarding information that resists translation". ${ }^{33}$ A more informative ethical analysis should consider two things: (i) empirical research that explores the actual accounts of people who are in some way connected with or concerned about $\mathrm{AD}$; and (ii) an assessment of how ordinary people (not just professionals) perceive, describe and recall genetic risk information.

The REVEAL study (Risk Evaluation and Education for Alzheimer's disease) was the first randomised controlled trial designed to evaluate the impact of susceptibility testing using ApoE $\varepsilon 4$. The study was conducted at four sites in the USA between 2004 and 2006. At one site, 162 asymptomatic adults who had an affected parent were randomly assigned either to receive or not receive susceptibility testing. Both groups were measured for symptoms of anxiety, depression and test-related distress six weeks, six months and one year after (non) disclosure. ${ }^{34}$ The results showed no significant differences between the two groups, although those who received a negative result showed significantly lower levels of test-related distress than did the positive group. ${ }^{35}$ A separate study compared the results of the REVEAL subjects with those who received monogenetic testing; ${ }^{36}$ subjects who learned that they were positive for the susceptibility gene experienced low levels of distress similar to those who tested positive for the monogenetic test. They also found that "both susceptibility and deterministic genetic testing appeared to be well-tolerated by using disclosure protocols that provided screening, education, counselling and follow-up". ${ }^{37}$ In the ethnographic arm of the study, Locke et al. raised questions about the relevance and comprehension of testing:

Among the majority of people whom we interviewed in this project, the late onset of the disease and the pressures of daily life, often involving care giving, combined with the inherent uncertainty of the scientific knowledge, encourage most people to set aside worries about what the future has in store for them.

We can draw a number of inferences from this preliminary data. It is widely recognised that epidemiological information has a small impact on risk perception because probabilistic information is not meaningful to people. People often simplify 
or condense complex information in order to retain the essence of its meaning. ${ }^{39}$ The characteristics that impress themselves upon risk perception are those which are linked to dread or which are already familiar to people. ${ }^{40}$ In the case of predictive testing for Mendelian disorders, the low uncertainty of information coupled with the genetically exceptional nature of the test produces high impact of risk perception and low recall error. For susceptibility testing for complex disorders, the high uncertainty of information coupled with information about lifestyle are likely to dampen the impact of risk perception, resulting in high recall error. There is no empirical evidence at this stage to suggest that susceptibility testing is psychosocially harmful, but the REVEAL study does confirm that receiving a positive result for either the rare or the common gene is 'well tolerated' when disclosure is mediated by genetic counselling.

As tests for multifactorial diseases become increasingly available to the public, it will become important to develop strategies for presenting risk information that can communicate increased levels of uncertainty. The emergence of the personal genomics industry and the marketisation of direct-to-consumer (DTC) genetic testing raise serious concerns that vendors do not acknowledge or communicate the uncertainty of probabilistic risk information. These concerns are especially relevant when DTC marketing blurs the distinction between 'medical diagnosis' and 'health information', between 'health prediction' and 'risk estimation'. For instance, McGuire et al. reported that approximately one-third of all respondents in a survey of social networking users considered the information obtained from personal genomics testing to be a medical diagnosis. ${ }^{41}$ Other studies have shown how techniques of argumentation, distraction and persuasion are employed in the promotion of prescription drugs. ${ }^{42}$ The major concern is that DTC advertising is more profitable when misleading the public about the effectiveness or safety of health-related products and services. By extension, selling genomic knowledge directly to consumers creates expectations about diagnosis, treatment and prevention which are, as yet, scientifically undeliverable. ${ }^{43}$

In the DTC model of susceptibility testing, the relationship between promise and complexity is configured in such a way that the uncertainty of probabilistic risk information does not seem to impair promises of 'prevention' and 'self-knowledge'; this is because the version of complexity they recruit in their marketing strategies is one that strips out the problem of non-linear or stochastic interactions between genes and environment. The uncertainty of risk information is merely 'complicated' (partial and incomplete) and assumes that risk profiles will become more accurate as more information is added over time. This, rather impoverished, version of complexity does not account for epistasis, pleiotropy and polygenicity.

\section{Conclusion}

At the beginning of this article, I argued that a dialectic of promise and complexity is perhaps a more suitable way of framing recent advances in genomic medicine. Placing the events of the last decade within the discursive poles of promise and complexity seeks to draw attention away from an overly pessimistic orientation based 
on profound disappointment. The completion of the Human Genome Project signalled both the widening of a new research agenda and the enlargement of promise as scientific inquiry oriented its attention to investigating the underlying biology of common complex diseases. In contrast to the unqualified promises made by politicians in 2000, a more circumspect account of progress is found among the accounts of the scientists themselves, wherein gene function and gene expression are described in terms of their sobering 'complexity'. The two case studies I have considered show that the problems of overcoming complexity of common diseases on the one hand, and sustaining promise on the other, require a systematic attempt to augment the supply of human DNA samples to increase statistical significance of large-scale research studies. It also shows the rather limited and controversial attempts to commercially extract promissory value from genomic data.

Whether we see the turn to complexity in the life sciences as a matter of discovery or of construction, the main issue is that scientists' uncertainty about the human genome demands an unprecedented volume of information pertaining to a whole population. This is not just information about a population but information for a population public goods that may improve health and prevent disease for future generations. In the last 10 years, we have seen the apparent difficulties of traditional bioethics in meeting these challenges of scale. The rapid global sharing of information is placing increasing pressure on scientists, administrators and ethicists to abandon principles based on individual autonomy (i.e. informed consent) and to adopt more flexible and universal principles based on notions of solidarity, reciprocity and collective responsibility. These issues have been sharply foregrounded in recent debates about the international 'harmonisation' of biobanks. On the one hand, there are pragmatic arguments that relaxing ethical governance to facilitate global flows of bioinformation and international collaboration will assist genomic research. ${ }^{44}$ On the other hand, there is a deep mistrust that we are witnessing the remobilisation of technoscientific power and that bioethics is incapable of matching this power. ${ }^{45}$

Perhaps the most significant advance in the last 10 years has been the explosion of genome-wide association studies (GWAS) and the ubiquity of the 'SNP' ${ }^{46}$ as the statistical unit of common genetic variation. GWAS have yielded highly robust information, identifying susceptibility alleles for common disorders such as diabetes, heart disease, Crohn's disease and several common cancers. However, the majority of risk factors discovered by this method make up only a moderate contribution to overall risk. ${ }^{47}$ Many markers have yet to be discovered, which points to what some have described as the 'missing heritability' of common complex diseases. ${ }^{48}$ Such knowledge gaps render the clinical application of genetic susceptibility testing suspect and limited. In the urban zone of personal genomics, there are concerns that the complexity of common diseases, or this missing heritability, will not be properly communicated to the consumer, especially when concealing uncertainty is more profitable. Ethical analysis must distinguish the difference between weakly and strongly predictive testing which, it has been argued, cannot be accomplished by ethical principles alone. If the promise of predicting common complex diseases is the future of genomic medicine, then understanding how people perceive, feel, remember, 
describe and communicate complex risk information should be on the agenda of future ELSI research.

\section{Acknowledgments}

The support of the Wellcome Trust and the Economic and Social Research Council (ESRC) is gratefully acknowledged. The work was part of the programme of Cesagen, the ESRC Research Centre for Economic and Social Aspects of Genomics.

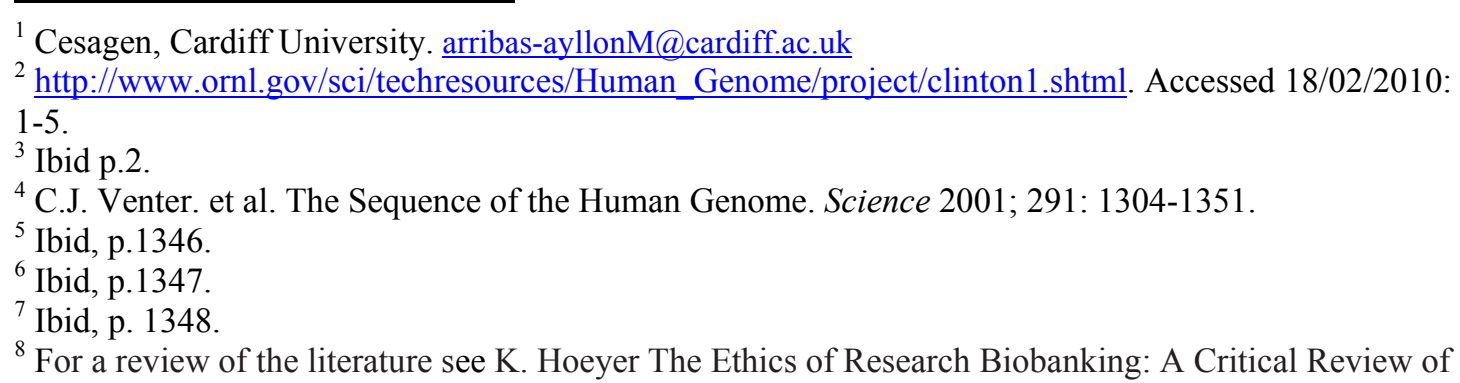
the Literature. Biotechnology and Genetic Engineering Ethics 2008; 25: 429-452. Also see R. Tutton. 2010. Biobanking: Social, Political and Ethical Aspects. In Encyclopedia of life sciences. John Wiley \& Sons, Ltd.

${ }^{9}$ P. Martin. Genetic Governance: The Risks, Oversight and Regulation of Genetic Databases in the UK. New Genetics and Society 2001; 20(2): 157-184. Also see A. Petersen. Biobanks" "Engagements": Engendering Trust or Engineering Consent? Genomics, Society and Policy 2007; 3(1): 31-43.

${ }^{10}$ A. Petersen. Securing our genetic health: engendering trust in UK Biobank. Sociology of Health and Illness 2006; 27(2): 271-292. Also see B. McNamara and A. Petersen. 2008. Framing Consent: The Politics of "Engagement" in an Australian Biobank Project. In Biobanks: Governance in Comparative Perspective. H. Gottweis and A. Peterson, eds. London: Routledge.

${ }^{11}$ Petersen op cit. note 9, p.37.

${ }^{12}$ B. Wynne. Public Engagement as a Means of Restoring Public Trust in Science - Hitting the Notes, but Missing the Music? Community Genetics 2006; 9: 211-220.

${ }^{13}$ G. Palsson. 2008. Anthropology and the New Genetics. Cambridge, NY: Cambridge University Press.

${ }^{14}$ R. Tutton, J. Kaye, and K. Hoeyer. Governing UK Biobank: the importance of ensuring public trust. Trends in Biotechnology 2004; 22(6):284-5.

${ }^{15}$ K. Berg. DNA Sampling and Banking in Clinical Genetics and Genetic Research. New Genetics and Society 2001; 20(1): 59-68.

${ }^{16}$ G. Williams. Bioethics and Large-Scale Biobanking: Individualistic Ethics and Collective Projects. Genomics, Society and Policy 2005; 1(2): 50-66.

${ }^{17}$ Tutton et al. op cit note 13, pp.284-285.

${ }^{18}$ B.M. Knoppers and R. Chadwick. Human Genetic Research: Emerging Trend in Ethics. Nature 2005; 6: 75-79.

${ }^{19}$ A. Tupasela. Locating Tissue Collections in Tissue Economies - Deriving Value from Biomedical Research. New Genetics and Society 2006; 25(1): 33-49. Also see H. Zwart. Challenges of MacroEthics: Bioethics and the Transformation of Knowledge Production. Bioethics Inquiry 2008; 5: 283293

${ }^{20}$ C. Waldby. 2000. The Visible Human Project: Informatic Bodies and Posthuman Medicine. London, New York: Routledge. Also see N. Rose. Neurochemical Selves. Society Nov/Dec 2003: 46-59.

${ }^{21}$ For a discussion of medico-legal issues see G. Laurie. 2002. Genetic Privacy: A Challenge to Medico-Legal Norms. Cambridge, NY: Cambridge University Press.

${ }^{22}$ T. Duster. Race and reification in science. Science 2005; 307 (18 Feb): 1050-51.

${ }^{23}$ N. Rose. 2007. The Politics of Life Itself: Biomedicine, Power and Subjectivity in the Twenty-First Century. New Jersey: Princeton University Press. p.161. 
${ }^{24}$ K.S. Rajan. 2006. Biocapital: The Constitution of Postgenomic Life. Durham: Duke University Press.

${ }^{25}$ M. Fortun. 2007 Promising Genomics: Iceland and DECODE Genetics in a World of Speculation. Berkely: University of California Press.

${ }^{26}$ A. Smart, R. Tutton, P. Martin, G.T.H. Ellison and R. Ashcroft. The Standardisation of Race and Ethnicity in Biomedical Science Editorials and UK Biobanks. Social Studies of Science 2008; 38(3): 407-423.

${ }^{27}$ Rose op cit note 22.

${ }^{28}$ P. Boddington and S. Hogben. Working Up Policy: The Use of Specific Disease Exemplars in

Formulating General Principles Governing Childhood Genetic Testing. Health Care Analysis 2006; 14 : $1-13$.

${ }^{29}$ C. Novas and N. Rose. Genetic Risk and the Birth of the Somatic Individual. Economy and Society 2000; 29(4): 485-513.

${ }^{30}$ E.H. Corder, A.H. Saunders, W.J. Strittmatter, D.E. Schemechel, P.C. Gasdkell, G.W. Small, A.D. Roses, J.L. Haines, and M.A. Perical-Vance. Gene Dose of Apolipoprotein E Type 4 Allele and the Risk of Alzheimer's Disease in Late Onset Families. Science 1993; 261(5123): 921-923.

${ }^{31}$ S.J. Roberts et al. Who Seeks Genetic Susceptibility Testing for Alzheimer's Disease? Findings from a Multisite, Randomized Clinical Trial. Genetics in Medicine 2004; 6(4): 197-203.

${ }^{32}$ H. Brodaty, M. Conneally, S. Gauthier, C. Jennings, A. Lennox, S. Lovestone. Consensus Statement on Predictive Testing for Alzheimer Disease. Alzheimer Disease Association Disorder 1995; 9: 182214; L.A. Farrer, M.F. Brin, L. Elsas, et al. Statement on the Use of Apoliopoprotein E Testing for Alzheimer Disease. JAMA 1995; 274: 1627-1629; S.G. Post, P.J. Whitehouse, R.H. Binstock, et al. The Clinical Introduction of Genetic Testing for Alzheimer Disease: An Ethical Perspective. JAMA 1997; 277: 832-836.

${ }^{33}$ J.H. Evans. A Sociological Account of the Growth of Principlism. Hastings Center Report 2000; 30(5) 31-38.

${ }^{34}$ R.C. Green, S.J. Roberts, A.L. Cupples, N.R. Relkin, P.J. Whitehouse, T. Brown, E.S. LaRusse, M. Butson, A.D. Sadovnick, K.A. Quaid, C. Chen, R. Cook-Deegan, L.A. Farrer. Disclosure of $A P O E$ Genotype for Risk of Alzheimer's Disease. The New England Journal of Medicine 2009; 361: 245-254. ${ }^{35}$ T.M. Marteau, S. Roberts, S. LaRusse, and R. Green. Predictive Genetic Testing for Alzheimer's Disease: Impact upon Risk Perception. Risk Analysis 2005; 25(2): 397-404.

${ }^{36}$ M.R. Cassidy, S.J. Roberts, T.D. Bird, E.J. Steinbart, A.L. Cupples, C.A. Chen, E. Linnenberger, and R.C. Green. Comparing Test-Specific Distress of Susceptibility Versus Deterministic Genetic Testing for Alzheimer's Disease. Alzheimer's \& Dementia 2008; 4: 406-413.

${ }^{37}$ Ibid p.410.

${ }^{38}$ M. Locke, J. Freeman, G. Chilibeck, B. Beveridge, and M. Padolsky. Susceptibility Genes and the Question of Embodied Identity. Medical Anthropology Quarterly 2007; 21(3): 256-276.

${ }^{39}$ A. Lippman-Hand, and F.C. Fraser. Genetic Counselling: Parents' Responses to Uncertainty. Birth Defects Original Article Series 1979; XV: 325-339.; E. Parsons and P. Atkinson. Lay Constructions of Genetic Risk. Sociology of health and Illness 1992; 14(4): 437-455.

${ }^{40}$ P. Slovic, B. Fischhoff and S. Lichenstein. 1980. Facts and fears: understanding perceived risk. In Societal Risk Assessment: How safe is safe enough? R.C. Schwing and W.A. Albers (eds.) New York: Plenum Press; S. Michie, J. Weinman, J. Miller, V. Collins, J. Halliday and T.M. Marteau. Predictive Genetic Testing: High Risk Expectations in the Face of Low Risk Information. Journal of Behavioural Medicine 2002; 25: 33-50.

${ }^{41}$ A.L. McGuire, C.M. Diaz, T. Wang and S.G. Hilsenbeck. Social Networkers' Attitudes Toward Direct-to-Consumer Personal Genome Testing. The American Journal of Bioethics 2009; 9(6-7): 3-10.

${ }^{42}$ S. Rubinelli. 'Ask your doctor'. Argumentation in Advertising of Prescription Medicines. Studies in Communication Sciences. Special Issue on Health Literacy 2005; 5 (2): 75-98; S. Rubinelli, K. Nakamoto, and P.J. Schulz. The Rabbit in the Hat: Dubious Argumentation and the Persuasive Effects of Prescription Drug Advertising (DTCA). Communication and Medicine 2008; 5(1): 49-58. S. Rubinelli, K. Nakamoto, J.P. Schulz and L. de Saussure. What Are We to Think About Consumer Advertising? A Case-Study in the Field of Misinterpreted Argumentation. Studies in Communication Sciences 2006; 6 (2): 337-348.

${ }^{43}$ M. Arribas-Ayllon, S. Sarangi and A. Clarke. Promissory Strategies of Personalisation in the Commercialisation of Genomic Knowledge. Communication and Medicine. Forthcoming. 
${ }^{44}$ S. Fortin and B.M. Knoppers. Secondary Uses of Personal Data for Population Research. Genomics, Society and Policy 2009; 5(1): 80-99.

${ }^{45}$ R.J. Karlsen, J.H. Solbakk and R. Strand. 2009. In the Ruins of Babel: Should Biobank Regulations be Harmonized? In The Ethics of research biobanking. J.H. Solbakk, S. Holm, B. Hofman, eds. Dordrecht: Springer.

${ }^{46}$ N. Risch and K. Merikangas. The Future of Genetic studies of Complex Human Diseases, Science 1996; 273: 1516-1517; D.G. Wang, et al. Large-Scale Identification, Mapping, and Genotyping of Single-Nucleotide Polymorphisms in the Human Genome, Science 1998; 280: 1077-1082; L. Kruglyak and D.A. Nickerson, Variation is the spice of life, Nature Genetics 2001; 27: 234-236; J.C. Stephens, J.A. Schneider, D.A. Tanguay, J. Choi, T. Acharya, S.E. Stanley. Haplotype variation and linkage disequilibrium in 313 human genes, Science 2001; 293: 489-493.

${ }^{47}$ G.W. Feero, A.E. Guttmacher and F.S. Collins. The Genome gets Personal - Almost. JAMA 2008; 299(11): 1351-1352.

${ }^{48}$ T.A. Manolio et al. Finding the Missing Heritability of Complex Diseases. Nature 2009; 461: 747753. 